\title{
Extended application of radon as a natural tracer in oil reservoirs
}

\author{
A.M.F. Pinto ${ }^{1}$, A.A. Barreto ${ }^{1}$, R.M. Moreira ${ }^{1}$ and E.B. Tambourgi ${ }^{2}$ \\ ${ }^{1}$ CDTN - Centre for Development of Nuclear Technology, PO Box -941, CEP: 30161-970, Brazil \\ ${ }^{2}$ UNICAMP - State University of Campinas, Av. Albert Einstein, 500 - CEP: 13083-852, Brazil
}

\begin{abstract}
In the 80's it was a common practice in the study of contamination by NAPL to incorporate a tracer to the medium to be studied. At that time the first applications focused on the use of ${ }^{222} \mathrm{Rn}$, a naturally occurring radioactive isotope as a natural tracer, appropriate for thermodynamics studies, geology and transport properties in thermal reservoirs. In 1993 the deficit of radon was used to spot and quantify the contamination by DNAPL under the surface. For the first time these studies showed that radon could be used as a partitioning tracer. A methodology that provides alternatives to quantify the oil volume stored in the porous space of oil reservoirs is under development at CDTN. The methodology here applied, widens up and adapts the knowledge acquired from the use of radon as a tracer to the studies aimed at assessing SOR. It is a postulation of this work that once the radon partition coefficient between oil and water is known, SOR will be determined considering the increased amount of radon in the water phase as compared to the amount initially existent as the reservoir is flooded with water. This paper will present a description of the apparatus used and some preliminary results of the experiments.
\end{abstract}

\section{INTRODUCTION}

The determination of residual oil saturation, SOR, has been used for a long time in order to model the performance of the reservoirs and predict its fall. In the seventies, the knowledge of SOR started to be used to identify remaining resources during the process of flooding. This knowledge was also important for recovering projects.

The quantity and the distribution of SOR are known to be critical parameters for maximizing production from an oil field. The estimation of such parameters is helpful to decide which method is more appropriate to the recovery and is supposed to be applied in order to obtain an economically viable production.

SOR varies widely according to the type of formation, with the properties of the oil and also with water properties. A generally accepted method to quantify the SOR is not known yet. Different measuring techniques are available in literature [1-3], but the results are not necessarily the same when applied to the same well. Such variations can be caused by the heterogeneity of the reservoir rock or even by the alterations caused in the reservoirs by the applied process of recovery. In the method proposed here, it is postulated that if the radon partition coefficient between the oil and the water is known, as the reservoir is flooded with water, it would be possible to determine the Residual Saturation by the increased quantity of radon in the aqueous phase in relation to the quantity initially present.

In this work, the methodologies that allow quantifying SOR in oil reservoirs are similar to the ones that use radon to monitor remediation of DNAPL in the contaminated areas [4-10]. These ideas were adapted and modified in order to try to develop a new method which can be able to estimate SOR in oil reservoirs by using natural radon present in the reservoir.

This methodology, which is being developed, is based on five main activities and will be tested in the field soon:

1. Knowledge of the area to be studied by sampling water, oil and porous rock medium.
2. Studying the porous rock medium in order to determine the radon concentration, porosity and permeability and some other characteristics.

3. Studying the fluids to determine, for instance, the densities, the salinities and also the partition coefficient of radon between water and oil.

4. Carrying out laboratory tests that involve the simulation of draining and imbibitions processes.

5. SOR determination.

The use of radon as a tracer in oil industries can become an alternative method that will allow a better evaluation of SOR during the whole useful life of the reservoir, enlarging the possibilities of choice and comparison between the methods available to a better evaluation of the reserves and consequently a more real planning of the oil recovery.

In this paper, a description of the experimental apparatus and the preliminary results will be presented.

\section{METHODOLOGY AND RESULTS}

The presence of natural radiation in oil fields is due mainly to the radioactive nuclides present in our planet since its formation. Their half-lives, $\mathrm{T}_{1 / 2}$, are long enough to allow their presence even today. This is the case of potassium- 40 $\left(\mathrm{T}_{1 / 2}=0.13 \times 10^{10}\right.$ years $)$, uranium-238 $\left(\mathrm{T}_{1 / 2}=0.45 \times\right.$ $10^{10}$ years $)$, uranium-235 $\left(\mathrm{T}_{1 / 2}=0.07 \times 10^{10}\right.$ years $)$ and thorium-232 $\left(\mathrm{T}_{1 / 2}=1.39 \times 10^{10}\right.$ years $)$.

Radon, whose atomic number is $\mathrm{Z}=86$, is produced in the earth's crust from the uranium decay. There are three naturally occurring radon isotopes: ${ }^{222} \mathrm{Rn}$ from the decay series of ${ }^{238} \mathrm{U},{ }^{220} \mathrm{Rn}$ from the decay series of ${ }^{232} \mathrm{Th}$ and ${ }^{219} \mathrm{Rn}$ from the decay series of ${ }^{235} \mathrm{U}$.

In the saturated and non saturated zones, ${ }^{222} \mathrm{Rn}$ is the most common due to its relatively long half-life (3.83 days) and also due to the fact that ${ }^{238} \mathrm{U}$ is the most abundant $(99.28 \%)$ among the uranium isotopes. The other two, ${ }^{219} \mathrm{Rn}$ and ${ }^{220} \mathrm{Rn}$, due to their half lives of $3.92 \mathrm{~s}$ and $54.5 \mathrm{~s}$ respectively, will not be considered in this study. 


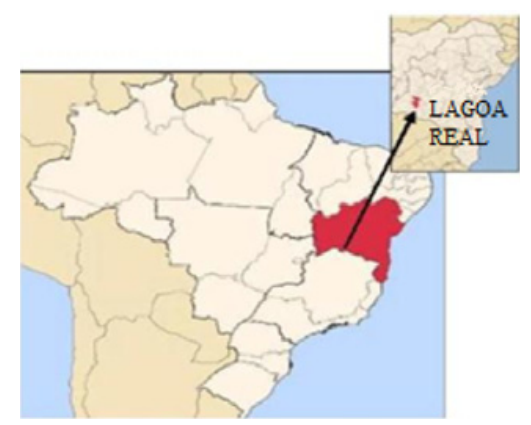

Figure 1. Map of Brazil, with the State of Bahia in detail, where one can see the Lagoa Real deposit.

Table 1. Density, salinity and conductivity of synthetic saline water.

\begin{tabular}{|c|c|c|}
\hline $\begin{array}{c}\text { Density } \\
\left(21^{\circ} \mathrm{C}\right)\end{array}$ & $\begin{array}{c}\text { Salinity } \\
\left(28^{\circ} \mathrm{C}\right)\end{array}$ & $\begin{array}{c}\text { Conductivity } \\
\left(28^{\circ} \mathrm{C}\right)\end{array}$ \\
\hline $1.0242 \mathrm{~g} / \mathrm{cm}^{3}$ & $69.2 \mathrm{~g} / \mathrm{L}$ & $67.1 \mathrm{mS} / \mathrm{cm}$ \\
\hline
\end{tabular}

Table 2. Density and viscosity of mineral oil.

\begin{tabular}{|c|c|}
\hline Density $\left(20^{\circ} \mathrm{C}\right)$ & $0.835 \mathrm{~g} / \mathrm{cm}^{3}$ \\
\hline viscosity $\left(37,8^{\circ} \mathrm{C}\right)$ & $75.2 \mathrm{SSU}$ \\
\hline
\end{tabular}

The tests were carried out in order to simulate the behavior of fluids in the oil reservoir and were done in the Tracer Testing Laboratory at CDTN. The core sample chosen to represent a medium with the characteristics of a reservoir was recovered from a uranium deposit which is rich in radium, generator of radon. The chosen deposit is the Uranium Province of Lagoa Real, the only active uranium deposit in Brazil and also in Latin America (Fig. 1).

The uranium mineral was crushed so that the particle sizes were below $1 \mathrm{~mm}$ in diameter. Next, the crushed material was sieved into the fractions Medium, Fine, and Very Fine according to the scale of Wentworth [11]. The choice for the dimension of the sand grains aimed at obtaining a porosity near $33 \%$.

The applied proportion was: $11.8 \%$ of the medium fraction, $11.8 \%$ of the very fine fraction and $76.4 \%$ of the fine fraction. The final composition was $1200 \mathrm{~g}$ of ore and $200 \mathrm{~g}$ of cement. The cement was added in order to help in the cohesion of the rock particles.

This mixture made possible to mould three core samples according to the requested dimensions: $15 \mathrm{~cm}$ height and $3.8 \mathrm{~cm}$ of diameter. When they were taken out from the mould in the laboratory, one of the core samples broke and was initially discarded (CP3).

The fluid used for flooding was synthetic saline water (Table 1) and the fluid to be displaced was simulated by a commercial mineral oil, EMCAplus 70, Table 2. Such fluids simulated the characteristics of the ones present in the reservoir.

In this study, the gaseous phase was not considered to cause any detrimental complexity to the laboratory experiments.
Table 3. Partition coefficient for radon distribution between mineral oil and water, and $\mathrm{n}$-hexan and water.

\begin{tabular}{|c|c|c|}
\hline & $\begin{array}{c}\text { Experimental } \\
\text { value } \\
\left(\mathbf{2 0}^{\circ} \mathbf{C}\right)\end{array}$ & $\begin{array}{c}\text { Theoretical } \\
\text { value } \\
\left(\mathbf{2 0}^{\circ} \mathbf{C}\right)\end{array}$ \\
\hline EMCAplus -70 & $39 \pm 2$ & \\
\hline n-hexan & $62 \pm 2$ & 58.1 \\
\hline
\end{tabular}

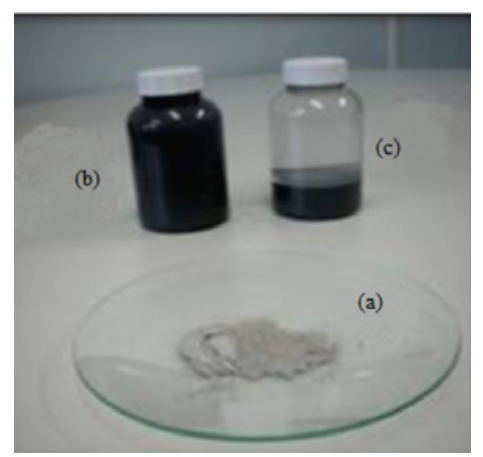

Figure 2. a) Dry powered sample b) wet powered sample without decanting c) wet powered sample after decanting.

\subsection{Determination of the partition coefficient}

The use of the tracer methodology for calculating the SOR was only possible due to the application of the partition concept presented by COOKE in 1971 [1].

Until 2003 no method was able to measure radon directly in the organic phase. Its value was indirectly calculated from the coefficient of solubility by Oswald [12].

The method chosen for its determination was the method proposed by CANTALOUB [13], which was based on the concept of multiple equilibria and sequential extraction. This method was chosen in order to allow a simple and direct measure of the radon partition coefficient distributed between an aqueous and organic phase.

The partition coefficient was determined before the beginning of the bench tests and the obtained result is presented in Table 3.

The partition coefficient for n-hexan was measured according to the method developed at CDTN as a way of checking this methodology. It was chosen because the theoretical coefficient was available from literature.

\subsection{Determination of the maximum activity of ${ }^{222} \mathrm{Rn}$}

The radon activity was first determined from the core sample which had been previously discarded. Part of the core sample 3 (CP3) was crushed in a jaw crusher and next powdered in a Powder Grinder with rim and socket of stainless steel, for 2 minutes, achieving a granulometry of approximately $400 \mathrm{Mesh}$ (Fig. 2a). Next, the powder was wet milled, for 6 hours and with a rotation of $600 \mathrm{rpm}$, in a kind of planetary mill, developed at CDTN [14].

The milling product, a suspension of rock in water at a concentration of $20 \%$ was distributed between bottle reservoirs b and c, (Fig. 2). After one day of decanting, the liquid phase presented crystalline characteristics (Fig. 2c). 


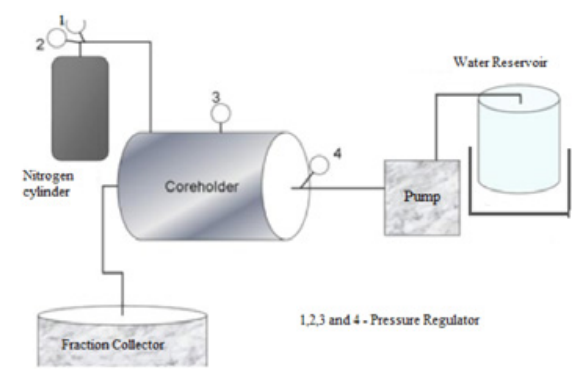

Figure 3. Mounting scheme used during the tests in porous medium.

The present radon concentration was determined by Liquid scintillation spectrometry, LSC, and the value found was $0.12 \pm 0.01 \mathrm{~Bq} / \mathrm{mL}$.

\subsection{Bench tests}

In order to perform the bench tests, a simulated reservoir set-up was constructed. Subsequently flooding experiments were performed.

In Figure 3, a sketch of the set-up used in the bench test for the study of the fluid dynamics of the water and oil through the core sample is presented.

In detail, the equipment consists of the following parts:

- The coreholder: container able to wrap the core samples under pressure. It consists of two compartments-an internal one, where the core sample stays closed in a Viton $\cap$ sleeve and where the water and oil flow and an external one, filled with a second water fluid or gas, which is able to keep the core sample under pressure.

- Gas supplying system composed of a cylinder and a pressure regulator. This cylinder is used to store $1.0 \mathrm{~m}^{3}$ of nitrogen at a filling pressure of $15 \mathrm{MPa}$. The regulator of the simple pressure stage is rated for a pressure at the entrance up to $20 \mathrm{MPa}$ and at the exit up to $15 \mathrm{MPa}$. The maximum flow is $100 \mathrm{~m}^{3} / \mathrm{h}$.

- Positive displacement pump model PU-2086 Plus, manufactured by Jasco, that causes a pressure that reaches up to $50 \mathrm{MPa}$. It can be programmed to give a constant flux from 0.001 to $20.00 \mathrm{~mL} / \mathrm{min}$.

- Manometers, whose maximal working pressure is $22.5 \mathrm{MPa}, 75 \%$ of the final value of the scale. This sensor was installed at the entrance of the coreholder in order to evaluate the load loss during the fluid passage through the core sample.

- Electronic balance Mark GEHAKA model BK 4400.

- Automatic sampler model U-200 manufactured by Eldex, programmable for time or for the number of drops. The capacity is 200 graduated vials of $15 \mathrm{~mL}$, acquired from PerkinElmer. These vials have covers, in case it is necessary to store the aliquots for some time before being analyzed.

- Stainless steel tubes, whose external diameter is $3.18 \mathrm{~mm}$ and the internal one is $2.10 \mathrm{~mm}$.

- Auxiliary connections from the firm Detroit (needle valves, three way valves).

During all experiments, the confining pressure registered at the exit of the gas cylinder was monitored in order to
Table 4. Core sample parameters.

\begin{tabular}{|l|l|l|}
\hline & CP1 & CP2 \\
\hline Diameters & $3.72 \mathrm{~cm}$ & $3.75 \mathrm{~cm}$ \\
\hline Height & $15.16 \mathrm{~cm}$ & $15.41 \mathrm{~cm}$ \\
\hline Mass & $295.21 \mathrm{~g}$ & $308.61 \mathrm{~g}$ \\
\hline Real Density & $2.79 \mathrm{~g} / \mathrm{cm}^{3}$ & $2.74 \mathrm{~g} / \mathrm{cm}^{3}$ \\
\hline Absolute Porosity & $35.72 \%$ & $33.64 \%$ \\
\hline
\end{tabular}

keep it at least $2 \mathrm{MPa}$ above the entrance pressure of the fluid in the coreholder. This value would guarantee that the water of injection would not pass between the rock core

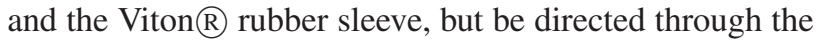
porous core itself.

The medium velocity of the fluid flow during the tests should be near the velocity value in oil reservoirs and was defined as $1.0 \mathrm{~cm} / \mathrm{h}$, a value near the value of $1.0 \mathrm{ft} / \mathrm{day}$, suggested by LAKE [15]. The fluid flow at the experiments was established at $0.06 \mathrm{~cm}^{3} / \mathrm{min}$.

The process of the reservoir formation was simulated by a drainage process followed by an imbibition process, in this order. In the oil engineering, this kind of testing reproduces the historical process of the formation of the reservoir rocks.

Drainage: During this process, the synthetic oil is injected into the reservoir in order to expel the water that is initially saturating the rock. In this step, the expelling of a wetting fluid by the non-wetting occurs. At the end of the drainage, the water volume remaining in the rock presents the irreducible water saturation or connate water.

Imbibition: In this step, the water is injected into the reservoir. The wetting fluid displaces the non-wetting fluid. At the end of the imbibition process, the oil volume that remains in the rock represents the residual oil saturation.

The concentration of ${ }^{222} \mathrm{Rn}$ was determined by LSC in the water and/or in the oil, when appropriate, at the exit of the coreholder. The used spectrometer, Quantulus ${ }^{\mathrm{TM}} 1220$, was manufactured by PerkinElmer.

Two of these tests already carried out are described. The first aimed at determining the maximal concentration of radon. The second one simulated the drainage process when the core CP1 was saturated with oil and the oil volume which was necessary for its saturation could be estimated.

The last test will represent the process of $\mathrm{CP} 1$ imbibition by injecting water and it is ongoing at the moment.

\subsubsection{First Bench Test}

Before beginning the core sample tests, the porous medium was characterized after saturation with synthetic saline water.

Table 4 presents the dimension of the core samples 1 and 2 and also the real density which is determined by helium gas picnometry. The absolute porosity was determined from the relation between the geometrical and real densities.

The first test aimed at determining the maximum activity of radon that would be used later for the calculation 


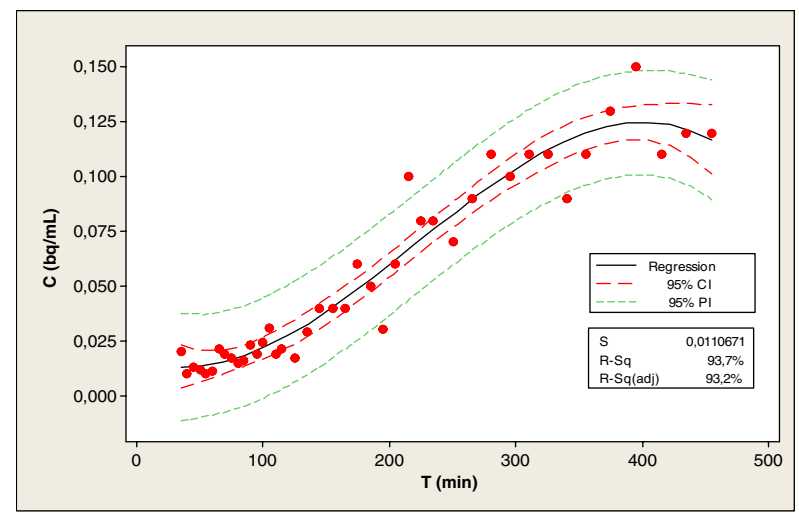

Figure 4. Results curve of radon activity as a function of time.

of SOR and it was determined before injecting oil for the first time in the core sample.

A core sample confining time of thirty three days warrants a ratio between the concentrations of radium and radon of $99.75 \%$. This was the minimum time considered appropriate in order to carry out the tests without jeopardizing the quality of the results and still keep the studies on a practical level.

$\mathrm{CP} 1$ was dismounted from the storing compartment. After removing the excess of water, $\mathrm{CP} 1$ was weighted and installed in the coreholder.

Before beginning the flooding tests, each of the sampling vials (micro tubes of polypropylene by Eppendorf) was filled with $1.20 \mathrm{~mL}$ of Optiphase Hisafe ${ }^{\mathrm{TM}}$ 3 cocktail and after that it was numbered and weighted.

The experiment consisted of a continuous injection of saline water at the entrance of the coreholder and its respective collection at the exit.

Samples were collected directly in the vials of polypropylene of $1.5 \mathrm{~mL}$ of nominal volume. A cap of the vial itself was used in order to minimize the radon losses during the collecting process. The time for filling each vial was 5 minutes. The predictable volume for the sampling was $0.3 \mathrm{~mL}$. The vial was completely filled with a total volume near $1.5 \mathrm{~mL}(1.20 \mathrm{~mL}$ of cocktail plus $0.3 \mathrm{~mL}$ of a sampled solution). Just after each collection, the vial was capped and agitated for approximately 30 seconds. The samples were then stored for a minimum period of 4 hours in order for radon to achieve secular equilibrium with its decay products before being analyzed.

After being analyzed, the vials were weighted again. The sampled volume was determined by difference of weight and the result of the analysis normalized this volume.

The total time of the sampling was of $8 \mathrm{~h}$. The results of the radon analysis given by the Radioanalytic Laboratory were plotted as shown in Figure 4.

In the Figure above, the following was noticed: the best fit trend line is a third degree polynomial. The equation given represents this curve and the standard deviation $\left(\mathrm{R}^{2}\right)$ associated to the fit. The maximum point of the polynomial was determined by setting the derivative of this equation to zero and solving for $\mathrm{X}$. The roots of this equation, $\mathrm{X} 1$ and $\mathrm{X} 2$, represent the points where the curve passes by the maximum and the minimum.

$$
\begin{aligned}
& X 1=38.41 \mathrm{~min} \quad Y=1.31 \times 10^{-2} \mathrm{~Bq} / \mathrm{mL} \\
& X 2=401.76 \mathrm{~min} \quad Y=1.25 \times 10^{-1} \mathrm{~Bq} / \mathrm{mL}
\end{aligned}
$$

The value of $Y=1.25 \times 10^{-1} \mathrm{~Bq} / \mathrm{mL}$ represents the maximum concentration found for $\mathrm{CP} 1$ according to the methodology applied.

This value was corroborated by the result $0.12 \pm$ $0.01 \mathrm{~Bq} / \mathrm{mL}$ determined from the milling of CP3.

\subsubsection{Second bench test}

In this experiment, the drainage process of the reservoir was simulated. Initially, the $\mathrm{CP} 1$ was taken from the storing compartment in which it was submerged, dried and weighted. It was then mounted inside the coreholder and the synthetic oil was injected continuously.

The water reservoir was replaced by an Erlenmeyer flask, which contained approximately $1000 \mathrm{~mL}$ of oil. The Erlenmeyer flask was weighted before and after the oil was injected. During the whole injection process, the Erlenmeyer remained on a mass balance. The line before the coreholder was also filled with oil.

As soon as the oil injection began, the sampling at the exit of the coreholder started as well. The vials, which were properly numbered and weighted, were replaced every hour. The collecting lasted for 72 hours.

The experiment was interrupted and the CP1 weighted and transferred to a closed compartment which contained oil. The final weight of the Erlenmeyer, containing the residual oil, was written down. The volume of the line before the coreholder was calculated. The collected vials were centrifugated in order to separate the oil from the water.

The oil volume which was necessary to saturate CP1 was calculated through the mass balance, that is, all the oil that entered in the in CP1 less the oil that left, represents the oil that remained in the CP1. The oil that entered was registered by the difference of weight of the Erlenmeyer flask before and after the experiment and the oil that left was calculated by the relation of the densities.

The total volume of the mixture, Vm, was directly read in the proper graduation of each vial. The volume of the mixture is composed of the collected water volume ( $\mathrm{Va}$ ) and the collected oil volume (Vo). We can assure that:

$$
\begin{gathered}
V m=V a+V o \\
M m=M a+M o \\
X a=V a / V m \\
X o=1-X a
\end{gathered}
$$

$X a$ is the fraction of water in the mixture and $X o$ is the fraction of oil in the mixture, Equation (2) can then be rewritten as:

$$
M m=\rho_{a} V a+\rho_{o} V o
$$




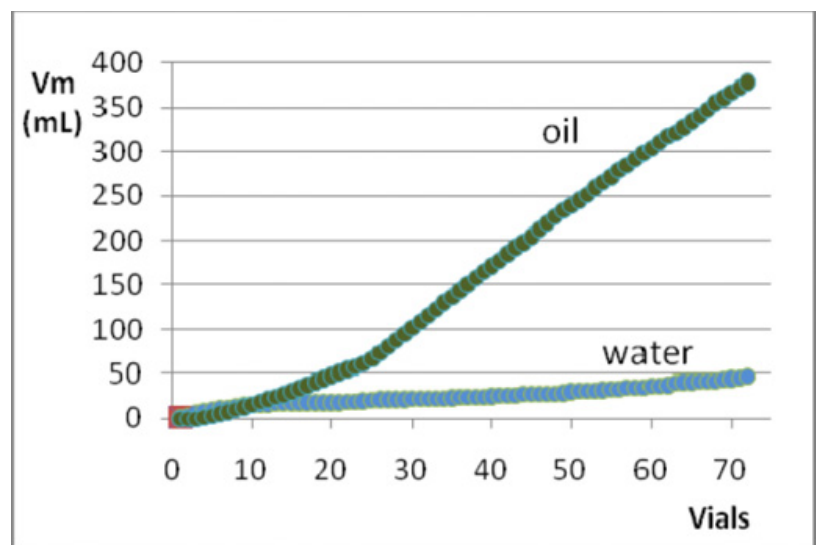

Figure 5. Result of accumulated water and oil expelled from CP1.

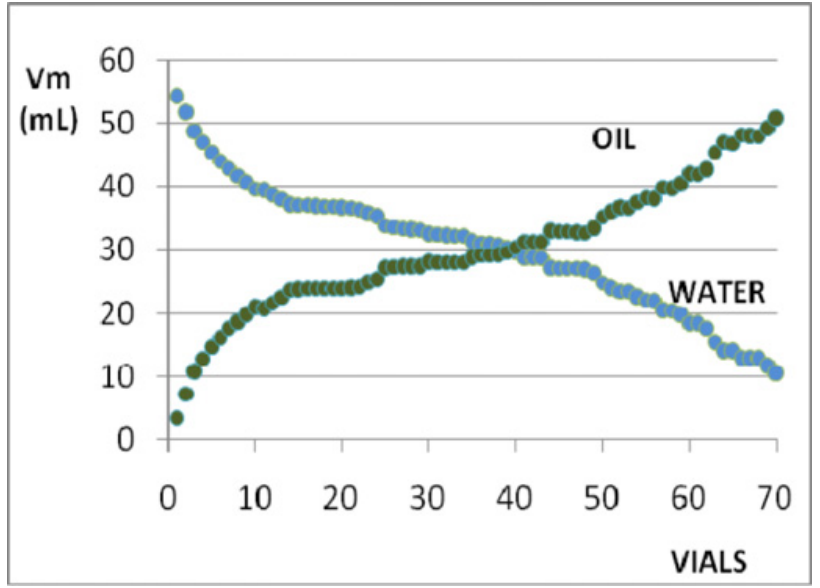

Figure 6. Accumulated result of the expelled water and of the oil that remained in $\mathrm{CP} 1$.

$$
\rho_{m} V m=\rho_{a} V m X a+\rho_{o} V m(1-X a)
$$

$X a$ and $X o$, for each vials, were calculated from Equations (4) and (6).

The obtained results allowed the mass balance to be derived in three different ways, which aimed at calculating the oil volume accumulated in CP1.

The weight registered by the balance in the beginning and also at the end of the experiment was used. The weight of the apparatus (Erlenmeyer and oil) before beginning the experiment was equal to $1562.8 \mathrm{~g}$ and at the end this weight had dropped to $1202.85 \mathrm{~g}$. The difference between these two weights divided by the oil density represents the oil volume that was injected into CP1 $(431.08 \mathrm{~mL})$. The oil that was expelled from CP1 was computed vial by vial. The accumulated volume of this oil was $378.10 \mathrm{~mL}$ and the water was $47.08 \mathrm{~mL}$ (Fig. 5). This means that CP1 was saturated with $52.98 \mathrm{~mL}$ of oil.

The saline water volume that remained in $\mathrm{CP} 1$ could be calculated by the difference between the water volume initially present and the volume occupied by the oil. This difference, $7.93 \mathrm{~mL}$, represents the irreducible water saturation $\left(S_{w i}\right)$ (Fig. 6).

\section{PRELIMINARY CONCLUSIONS}

The work presented here is still in progress.

The main objective of this work is to prove the viability of the proposed technique for oil reservoir. The results obtained until now have not shown any showstoppers and that the chosen way is promising.

The author recognizes that even if the proposed technique seems viable, there is a long way ahead which involves analytical development and real field tests.

In addition, a number of laboratory flooding experiments remain to be carried out. Therefore, this text may be regarded as a progress report only, and final conclusions on the applicability of the proposed technique is not possible for the time being.

\section{References}

[1] Cooke, C.E. Jr.: Method of Determining Residual Oil Saturation in Reservoirs, U.S. Patent 3590 923, Exxon Production Research Co. (July 6, 1971)

[2] Deans, H.A. and Majoros, S.: The Single Well Chemical Tracer Method for Measuring Residual Oil Saturation, Final report, DOE/BC20006-18, (October 1980)

[3] Tang, J.S., and Harker, B.C., Interwell Tracer Test to Determine Residual Oil Saturation in a Gas-Saturated Reservoir; Part I: theory and design; JCPT, 30, $\mathrm{n}^{\circ} 3$, p. 76-85 (May-June 1991)

[4] Minquan, Jin. et al.: Partitioning Tracer Test for Detection, Estimation and Remediation Performance Assessment of Subsurface Nonaqueous Phase Liquids, Water Resources Research, 31, n ${ }^{\circ}$, p. 1201-1211 (1995)

[5] Wilson, R.D. and Markey, D.M.: Direct Detection of Residual Nonaqueous Phase Liquid in the Saturated Zone using SF6 as a Partitioning Tracer. Environmental Science \& Technology, 29: p. 1255-1258 (1995)

[6] Nicole T.N. and Brusseau, M.L.: Field Study of the Partitioning Tracer Method for Detection of Dense Nonaqueous Phase Liquid in a Trichloroethenecontaminate Aquifer. Environmental Science \& Technology, 30: p. 2859-2863 (1996)

[7] Semprini, L.: Modeling and Field Studies of Radon222 in Geothermal Reservoirs. Ph.D. Dissertation, Stanford University (1986)

[8] Hopkins, O.S.; Radon-222 as an Indicator for Nonaqueous Phase Liquids in the Saturated Zone: Developing a Detection Technology. Master's Thesis, Oregon State University (1994)

[9] Gottipati, S.: Radon-222 as a Tracer for Performance Assessment of NAPL Remediation Technologies in the Saturated Zone: an Experimental Investigation. Master's Thesis, Oregon State University (1996)

[10] Hunkeler, D., E. Hoenh, P. Höhener, and J. Zeyer: ${ }^{222} \mathrm{Rn}$ as a Partitioning Tracer to Detect Diesel Fuel Contamination in aquifers: Laboratory Study and Field Observations. Environmental Science \& Technology, 31: p. 3180-3187 (1997) 
[11] Wentworth C.K.: A scale of grade and class terms for clastic sediments. J. of Geology, 30: p. 377-392 (1922)

[12] Clever, L.H. Solubility data series, volume 2: Krypton, Xenon and Radon- gas solubility; International Union of Pure and Applied Chemistry; Pergamon Press; Oxford/UK (1979)

[13] Cantaloub, M., J. Higginbotham, and L. Semprini The Determination of Rn Partition Coefficients for Several Liquid Scintillation Cocktails 43th Annual
Conference on Bioassay, Analytical, and Environmental Radiochemistry, Charleston, SC November 09-11 (1997)

[14] Leal, J.M. Desenvolvimento da Tecnologia do Pó de Turmalina. Ph.D. Dissertation, Federal University of Ouro Preto (2008)

[15] Lake, L.W. The Society of Petroleum Engineers Reservoir Characterization Course (1998) http:// www . spe . org/web/training/demo/00frame. htm15/04/2010 